\title{
Laminated Structures and Measurement of Magnetostriction for Transducer Applications
}

\author{
Cao Qinghua ${ }^{1,2,3}$, Chen Dingfang ${ }^{2}$, Lu Quanguo ${ }^{*}, 3$, Yan Jianwu ${ }^{3}$, Tang Gang ${ }^{3}$, Zhu Zhifang ${ }^{3}$, \\ Zhang Xiaoxing ${ }^{3}$ and Tao Zhen ${ }^{3}$ \\ ${ }^{1}$ School of Automation, Wuhan University of Technology, Wuhan, 430083, China \\ ${ }^{2}$ Institute of Intelligent Manufacturing and Control, Wuhan University of Technology, Wuhan, 430063, China \\ ${ }^{3}$ Institute of Micro/nano Actuation and Control, Nanchang Institute of Technology, Nanchang, 330099, China
}

\begin{abstract}
This paper aims to designed a prototype for achieving the full magnetostriction of Galfenol, Lamination struction of transducer is designed and the radiation impedance is expressed in mechanical terms, a simplified onedimensional modeling of SPICE magnetostrictive transducer is established, the electrical input impedance is simulated as the function of frequency and experimentally adjusting the values is to match an existing impedance curve. a good fit between model and measurement is shown by measured and simulated electrical input impedances for the device.
\end{abstract}

Keywords: Dynamic system, laminated beam, magnetostriction, transducer.

\section{INTRODUCTION}

Magnetostriction is the change in a material's dimensions resulting from a change in its magnetic state, or vice versa. The deformation is extremely small, with strains on the order of tens of parts-per-million in elemental magnetostrictives and over a thousand of parts-per-million for giant magnetostrictives [1]. In most applications, the change in shape is too small to be observed with the naked eye or detected through touch. Magnetostrictive gallium-iron alloys (collectively known as Galfenol) chosen as a candidate active material. One of the remarkable features of Galfenol are its competitive strain capability, attractive magnetic permeability, and mechanical strength. The last characteristic is particularly significant as it allows access to a design space inaccessible to other high-strain active materials, both magnetostrictive and piezoelectric [2].

This paper aims to demonstrate a design approach that takes advantage of all Galfenol's unique properties in a way that is easy to design, simple to build, and general enough to apply to a wide range of scenarios. We have used a unidimensional design model for producing the prototype design while the results have been corroborated with threedimensional, nonlinear finite element analysis. The fabrication of the device is accomplished by assembling thin layers of Galfenol steel into two laminated structures, winding drive coils on these structures, and placing permanent magnets between them. Finally, We have developed a full uni-dimensional and multi-domain model for simulating the performance of the device [3]. Designed a

*Address correspondence to this author at the Intertek Hi-tech Development Zone, Tianxiang Road, No. 189, Nanchang, Postcard: 330099, China;

Tel: 13672218765; E-mail: qh9863@126.com prototype drive with the goal of achieving the full magnetostriction of Galfenol; the design was completed with a simplified one-dimensional model and verified with finite element analysis. Simulated the performance of the drive with one-dimensional modeling that includes the magnetic domain and eddy current losses [4]. A configurable Galfenol drive concept is proposed and investigated in this dissertation. The goal of this project is to lower the design barriers associated with magnetostrictive transducers by introducing a exible drive that is configured with a set of simplified design tools. The attractive material properties of Galfenol are successfully exploited: 1) the material's full strain capability is capable of being fully accessed 2) the mechanical strength eliminates the need for a stress-bolt (although diffculty with endmass bonding has been found to be a related issue), and 3) the high permeability has been used to create a low-leakage circuit that allows the 1TDC bias to be achieved with permanent magnets located away from the drive segment. In addition, this design achieves the necessary features for magnetostrictive design without resorting to auxiliary structures [5-7]. Conclusions from this study are divided into three groups that deal with the material, the modeling, and the prototype drive.SPICE modeling of magnetostrictive devices in which the magnetic domain and eddy current losses are included. The remanent induction, $\mathrm{Br}$, of the permanent magnets appeared to be less than the value speci ed by the manufacturer. A good fit between model and measurement was observed for the case that the remanence value used in the model was $75 \%$ of the manufacturer's specified remanence flux density. The reason for this discrepancy is unknown and it is suggested that future efforts experimentally verify the remanent induction of permanent magnets before assembling them into a device $[4,8,9]$. 


\section{TRANSDUCER OF MAGNETOSTRICTIVE}

Modeling is the process of using a mathematical framework to predict the behavior of a physical system. modeling is used to simulate the performance of electroacoustic devices. An effective scheme to mitigate eddy current losses is to divide the cross-section of the conductor (as seen by the magnetic flux) into several smaller conductors, each electrically insulated from the others. A key requirement for these sub-conductors is that the smallest dimension be less than the penetration depth for the maximum frequency of interest. Another way this is practically implemented is by slicing the conductor into very thin sheets and reassembling it with electrical insulation between the sheets. This process is known as lamination and the sheets are called lamina. When reassembled, only a portion of the cross-sectional area is actually made up of the conductive metal; the rest is taken up by insulation and epoxy. This gives rise to a common metric in laminated structures, the stacking factor, which is the ratio of the conductive metal area in the cross-section to the total crosssectional area. In practice, stacking factors between 0.85 and 0.99 are common. Lower values are generally indicative of thinner laminations that require more epoxy layers.

Instead of slicing the lamina from a solid piece, it is usually more economical to first roll the material into thin sheets and then punch laminations from the sheet and stack the laminations to achieve the nal structure. This, however, is not always possible due to limitations of certain materials. The most prevalent use of laminated structures is in electrical transformer cores and electric motor assemblies. The bulk of the literature on laminated structures is therefore to be found in the electrical engineering field and not in reference to magnetostrictive transducers. wryly described electrostatic loudspeakers as being Imerely a noisy capacitor," sometimes it is useful to think of the laminated Galfenol structures presented in this dissertation as merely being noisy transformer cores.

An important acoustical effect to consider in transducer design is the radiation impedance on the radiating face. When a piston moves against a fluid, it experiences a complex radiation impedance. Mathematically, the acoustical radiation impedance of a rigid, baffled, circular piston is calculated as

$Z_{\text {rad }}^{a}=\frac{\rho_{0} c_{0}}{A}\left[R_{1}(2 k a)+j X_{1}(2 k a)\right]$

where

$$
\begin{aligned}
& R_{1}(2 k a)=1-\frac{2 J_{1}(2 k a)}{(2 k a)} \\
& X_{1}(2 k a)=\frac{2 H_{1}(2 k a)}{(2 k a)}
\end{aligned}
$$

$k$ is the wave number, $a$ is the piston radius, $A$ is area of the piston face, $j=\sqrt{-1}$ is an imaginary number, and $\rho_{0}$ and $c_{0}$ are the density and sound speed of the fluid, respectively. $J_{1}$ is the first order Bessel function and $H_{1}$ is the first order Struve function. The quantity $k a$ (sometimes called the
Helmholtz number) is a dimensionless number that effectively compares the size of the piston to the frequency at which it is radiating. $R_{1}$ and $X_{1}$ are the real and imaginary parts of the normalized radiation impedance and are plotted in Fig. (1). Note that for large values of $k a, X_{1}$ goes to zero and $R_{1}$ approaches unity.

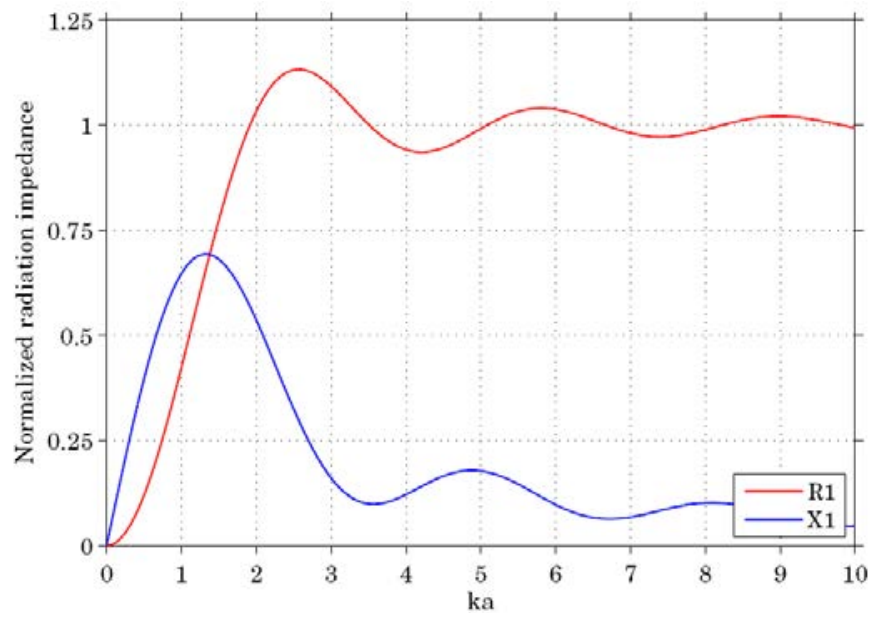

Fig. (1). Normalized real and imaginary parts of the radiation impedance for a baffled, circular piston.

It is important to note that the radiation impedance is often expressed in mechanical terms as the mechanical radiation impedance, $Z_{\text {rad }}^{m}$, where

$Z_{\text {rad }}^{m}=Z_{\text {rad }}^{a} A^{2}$

This is simply a transformation of the impedance quantity from the acoustical to mechanical domain.

\section{ONE-DIMENSIONAL DEVICE MODELING}

With two material descriptions in hand, it is now appropriate to consider the modeling of devices based on the magnetostrictive effect. Even devices built primarily out of magnetostrictive material, require careful device-level modeling. A basic, but useful, modeling technique, onedimensional modeling, is now presented.

One-dimensional modeling radically simplifies the description of a system by considering wave-propagation in only one direction. Essentially, this model discards the full device geometry and describes the system in terms of propagation distances. The actual utility of this method depends on the system being modeled, but for devices operated longitudinally (such as tonpilz transducers), it forms a good description. As is demonstrated with some of the magnetics modeling in this dissertation, one-dimensional models also work well for describing single variable systems that are not necessarily constrained to a single space dimension.

In transduction, the dynamic solution is of primary concern, but in some cases, especially in magnetism, the static solution is critical as it describes the conditions under which the dynamic problem takes place. Dynamic systems are characterized by having a time dependence. To illustrate, two basic dynamic systems are shown here: one mechanical and one electrical. 
A cornerstone concept for understanding mechanical vibrations (and by extension, acoustic oscillation) is the idea of a forced, damped, simple harmonic oscillator. This system consists of a sinusoidal force of magnitude $\mathrm{F}$ applied to a point mass, $m$, situated on a linear spring of stiffness, $k_{m}$, and a dashpot with mechanical resistance (damping), $R_{m}$. A reference point is established by fixing one end of the spring and dashpot. All elements in this system are considered to be ideal, not having the characteristics of the other elements.

However, this term may also accurately describe electrical systems with time-varying behavior. An example of a dynamic system $i \mathrm{n}$ the electrical domain is the basic $L R C$ circuit, so named the circuit is solely comprised of an inductance, $L$, a resistance, $R_{e}$, and a capacitance, $C_{e}$ energized by a sinusoidal voltage of magnitude $V$. Fig. (2) shows this circuit. The ground symbol is used to establish an electrical reference point. Again, all elements are considered to be ideal. Kircho's loop law for the $L R C$ circuit is:

$V=L \frac{d i}{d t}+R_{e} i+\frac{1}{C_{e}} \int i d t$

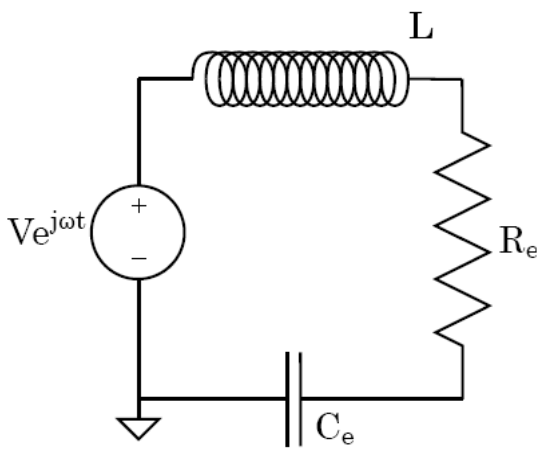

Fig. (2). An $L R C$ circuit.

The most fundamental concept in analogy is that energy exists in each physical domain and that the transfer of this energy can be characterized by two variables: an effort variable, e, and a flow variable, $f$. Alternatively, these variables are also called the across and through variables. It is convenient to require all domains to handle energy in a consistent manner; therefore, the standard requirement is that the product of the effort and flow variables have units of power (watts in this document). Assigning physical meaning to the effort and flow variables such that the ratio of effort to flow results in an impedance quantity is called an impedance analogy. In some cases, models are simplified by designating the effort and flow variables such that their ratio is an admittance quantity; this is known as the mobility analogy. For clarity, all circuits in this document are presented in the impedance analogy.

Equations (5) may be rewritten in the generalized form:

$e=I \frac{d f}{d t}+R f+\frac{1}{C} \int f d t$

Both these systems include all three passive (unpowered) elements: the inertance, $I$, describes the derivate term; the resistance, $R$, defines the proportional term; and the compliance, $C$, details the integral term. In true analogies, inertances and compliances represent energy storage while resistances indicate energy dissipation. The SI impedanceanalogy variables for the electrical, mechanical along with their units and the circuit symbols used in this document.

It is clear that systems combining these three elements are described by differential equations. Conversely, it is possible to use these elements to represent first and secondorder differential equations.

\section{ONE-DIMENSIONAL MODELING EXAMPLE}

The use of one-dimensional modeling is illustrated here for the HUSL Galfenol transducer This transducer is an good choice for illustrating these techniques because it includes physics from electricity, magnetism, mechanics, and acoustics.

Many properties in this model were measured from the device. All dimensions were determined with a caliper. A stereoscope was used to count the number of laminations and coil turns. The DC resistance of the coils was measured with a multimeter. Values for Galfenol's $Q_{m}, s_{33}^{H}, d_{33}$ and $\mu_{33}$ were taken from Wilson's book. Other material properties are from widely available sources.

All values in the model are stored as parameters using .param statements. SPICE recognizes standard SI unit prefixes as value modifiers that expression must be flagged with curly braces. Notice that this rule also holds for circuit component values.

Simulating the device as a projector, the excitation comes from the electrical domain (thinnermost loop). Here a $1 \mathrm{~A}$ current source is used in order to provide easy access to the electrical input impedance (simply the voltage at node $Z$ ). Also modeled in the electrical domain is the electrical resistance, $R_{\text {coil }}$, of both drive coils. These coils are wound in both electrical and magnetic series.

Wrapped around the electrical domain is the magnetic reluctance domain. Coupling is achieved with the timedifferentiating gyrator In this domain there are three types of magnetic impedances: lossy reluctances included in the piezomagnetic pieces, lossy reluctances representing the laminated circuit and the unlaminated magnet, and leakage reluctances. The goal of the magnetic circuit design is to deliver magnetic energy to the piezomagnetic piece that couples the reluctance domain into the mechanical domain.

The mechanical domain is intuitive because it pictures the actual device geometry: two drive sections that are situated between a headmass and a tailmass. Two material block pieces are used to model the tailmass and the magnet is modeled as a point mass. Mechanical losses, $R_{m}$, are calculated for this domain from the mechanical quality factor of Galfenol with some simplifying assumptions.

Acoustically, only two elements are used: the radiation impedance on the radiating face and the end face of the tailmass. Rather than add two more domains to the model, these impedances have been translated into the mechanical domain with equation 4 . Also, the radiation impedance has been simplified to be the characteristic impedance of 
propagating fluid, $Z_{0}=\rho c$, which, when multiplied by the radiator area, is equivalent to the mechanical radiation resistance, $R_{\text {rad }}$, at high $k a$ values. For this model in which the transducer is considered to be in air, the radiation impedance is very small and can be overlooked without much error. It cannot be neglected, however, when modeling the transducer in-water because the characteristic impedance of water is approximately 3,500 times the characteristic impedance of air.

To compare the model and measurement, the electrical input impedance is simulated as a function of frequency. This is accomplished with an AC analysis consisting of a 2000 -point linear frequency sweep between $1 \mathrm{kHz}$ to $100 \mathrm{kHz}$.

Both measured and simulated electrical input impedances for the device are shown in Fig. (3). The impedance was measured on an HP 8194 impedance analyzer. Notice that the model is not perfect, but it is in good agreement with the model and includes the major features. The inductance in the magnetic circuit is indicated by the slope of the impedance. First and second longitudinal resonances are reasonably well-predicted (within $10 \%$ of the actual value) and the effective coupling is similar (indicated by the separation of the first resonance and anti-resonance frequencies). The $90^{\circ}$ phase angle is indicative of the inductive nature of the device. As frequency increases, however, the complex eddy current losses increase, pulling the phase angle down toward $45^{\circ}$ a characteristic of fully-formed eddy currents.
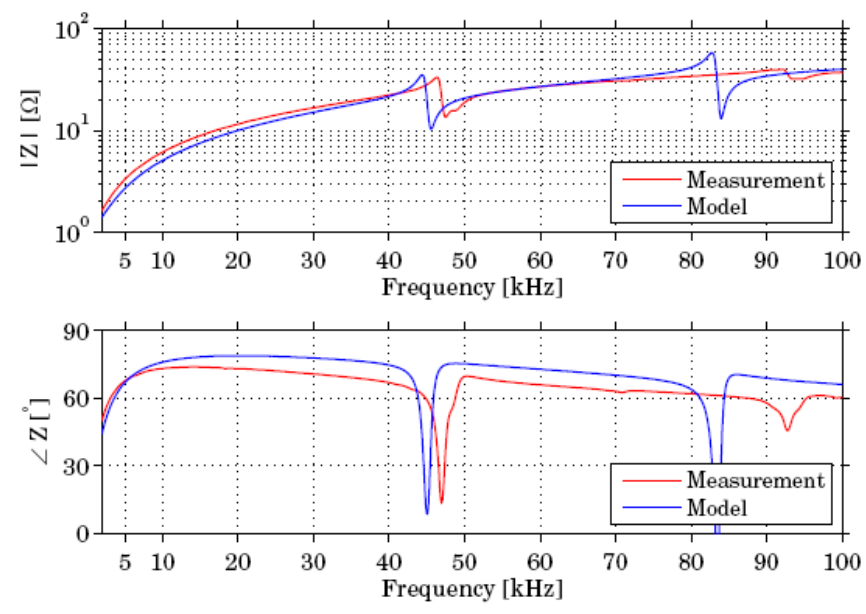

Fig. (3). Electrical input impedance measurement of the HUSL Galfenol transducer compared to the simulation of the onedimensional model.

The one-dimensional model shown in Fig. (4) is quite complex and it is worth noting that much simpler models may be used to achieve very similar behavior. With appropriate values for these four circuit components, the simpli_ed model can produce very similar results to the full one-dimensional model of Fig. (4). A comparison of the simple model to the full model is presented in Fig. (5) Even though the model has been significantly reduced in complexity, many important features are still resolved. One practical use of this model is to create simple electrical circuits that simulate the electrical load of real transducers for testing purposes. Notice, however, that in the simplified model only the first resonance is captured and that the phase is an ideal $+90^{\circ}$ away from resonance.

The real drawback to the simplified model lies in determining appropriate values for the four components. Experimentally adjusting the values to match an existing impedance curve is relatively straightforward, but calculating these component values from basic measurements is a much more daunting task. This is one of the advantages to the full model: many such calculations are included so that the inputs do not need to be effective values for the whole system. Similarly, the outputs of the full model are much more accessible; extracting certain quantities from the simplified model would also require involved calculations.

This leads to considering the two models in light of their intended use. While the simplified model can be used to quickly form a rough model of an existing transducer, it would be a very poor choice for design work. The full model, however, it intended to be used for this purpose and a major reason for its complexity is simplifying the design process by working with design variables.

With so many components, the most important circuit elements in the full model are easily obscured. There are two main drivers of the impedance in the full model: first, the magnetic reluctances model the inductive rise and phase droop and secondly the lossy piezomagnetic bar pieces resolve the resonant features.

\section{CONCLUSION}

This paper has examined the Galfenol configurable drive from initial concept to in-water testing of a prototype device. The present chapter reviews these efforts, makes some conclusions regarding the outcomes of the study, discusses the contributions made by this project, recommends areas for improvement, and suggests topics for future work.

Designed a prototype drive with the goal of achieving the full magnetostriction of Galfenol; the design was completed with a simplified one-dimensional model and verified with finite element analysis.

Simulated the performance of the drive with onedimensional modeling that includes the magnetic domain and eddy current losses. A configurable Galfenol drive concept is proposed and investigated in this dissertation. The goal of this project is to lower the design barriers associated with magnetostrictive transducers by introducing a exible drive that is configured with a set of simplified design tools. The attractive material properties of Galfenol are successfully exploited: 1) the material's full strain capability is capable of being fully accessed 2) the mechanical strength eliminates the need for a stress-bolt (although diffculty with endmass bonding has been found to be a related issue), and 3) the high permeability has been used to create a low-leakage circuit that allows the 1TDC bias to be achieved with permanent magnets located away from the drive segment. In addition, this design achieves the necessary features for magnetostrictive design without resorting to auxiliary structures. Conclusions from this study are divided into three groups that deal with the material, the modeling, and the prototype drive. 


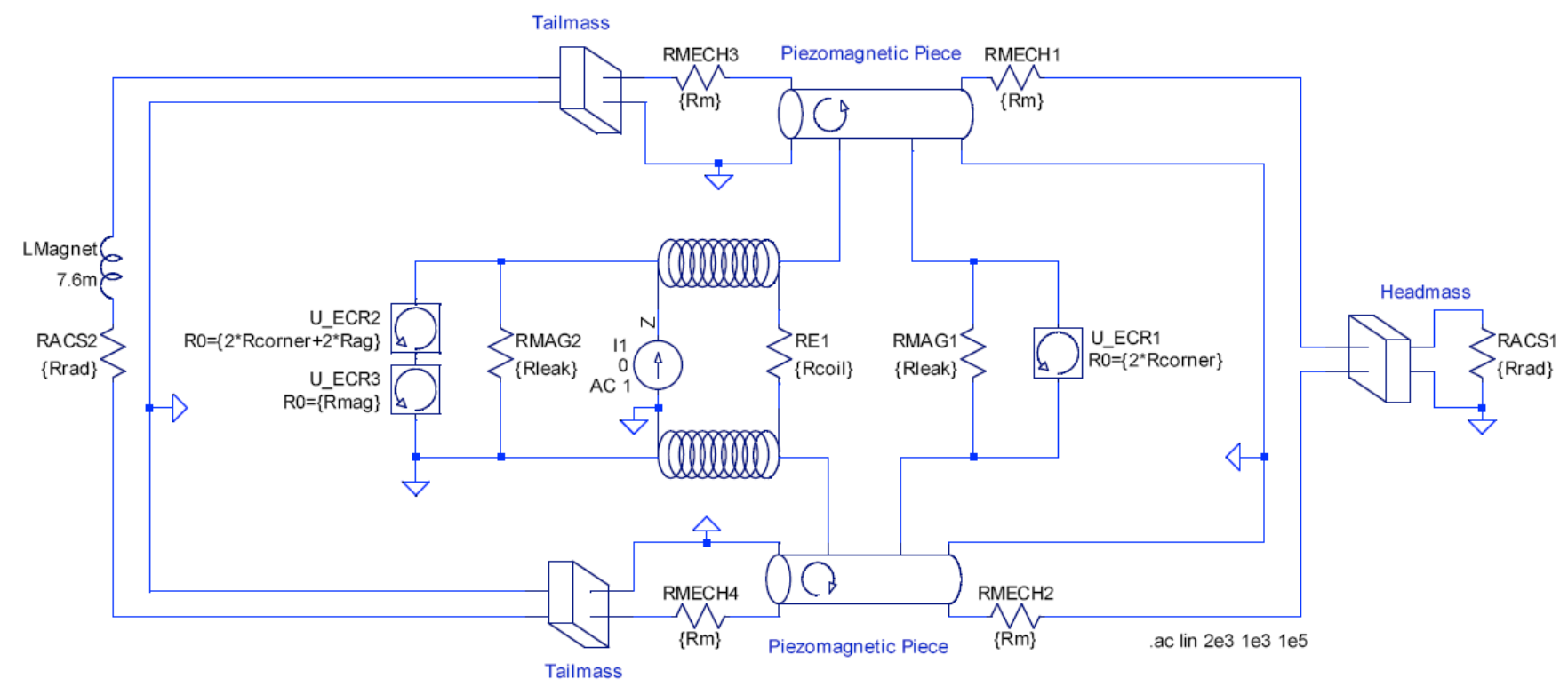

Fig. (4). One-dimensional model of the HUSL Galfenol transducer in SPICE.
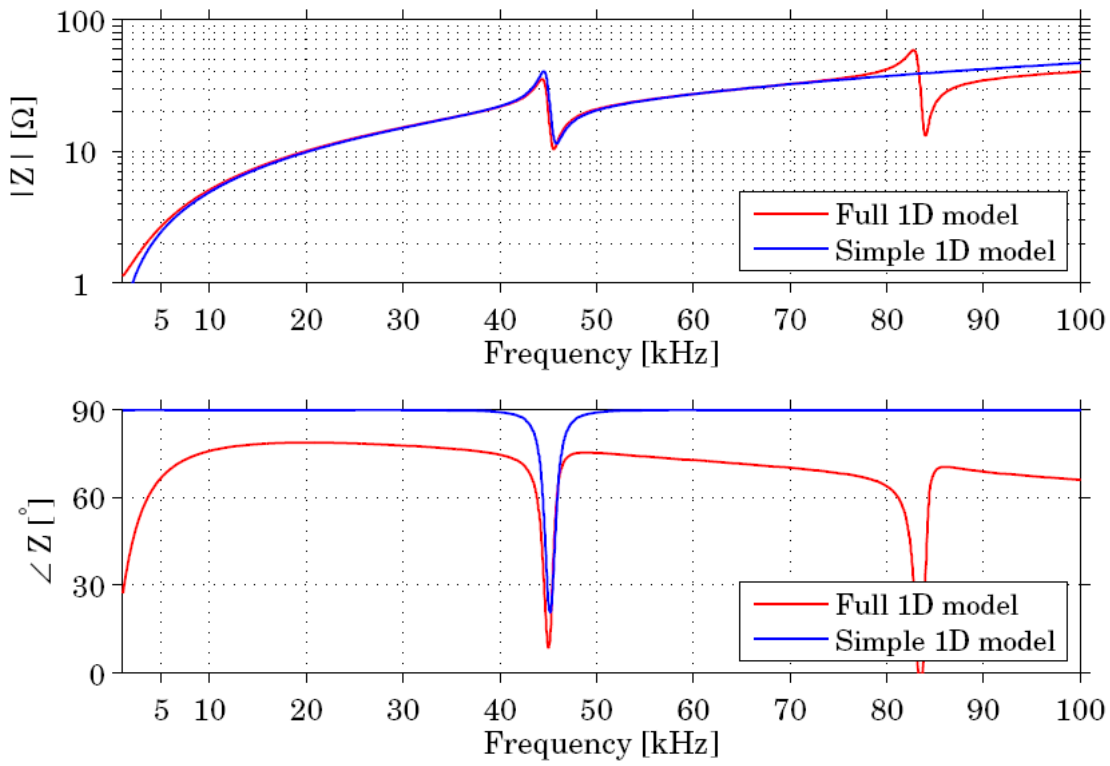

Fig. (5). Simulated electrical input impedance of the the Galfenol transducer for the full and simple circuit models.

SPICE modeling of magnetostrictive devices in which the magnetic domain and eddy current losses are included. The remanent induction, $\mathrm{Br}$, of the permanent magnets appeared to be less than the value specified by the manufacturer. A good fit between model and measurement was observed for the case that the remanence value used in the model was $75 \%$ of the manufacturer's specified remanence flux density. The reason for this discrepancy is unknown and it is suggested that future efforts experimentally verify the remanent induction of permanent magnets before assembling them into a device.

\section{CONFLICT OF INTEREST}

The authors confirm that this article content has no conflict of interest.

\section{ACKNOWLEDGEMENTS}

The authors would like to acknowledge the financial support by the National Natural Science Fund of China (Grant No. 51165035, 51175395 and 51161019), and Youth Science Fund of Jiangxi province office of education of China (Grant No. GJJ11247).

\section{REFERENCES}

[1] M. Engelhardt. LT spice Switcher CAD IV, Linear Technology Corporation, 2010 .

[2] G. Engdahl. Handbook of Giant Magnetostrictive Materials, Electromagnetism, Academic Press, New York, NY, 2000.

[3] M. Velaquez. Analysis of the E ective Coupling Coe cient of a Terfenol-D Tonpilz Transducer, Master's thesis, The Pennsylvania State University, State College, PA, 2005. 
[4] S. P. Porter, and S. C. Thompson. A Preliminary SPICE Model to Calculate the Radiation Impedance of a Ba ed Circular Piston," in Proceedings of the 27th Convention of the Audio Engineering Society, New York NY, USA,2009.

[5] B. D. Cullity, and C. D. Graham. Introduction To Magnetic Materials, second ed., Wiley, Hoboken, New Jersey, 2009.

[6] C. H. Sherman, and J. L. Butler. Transducers and Arrays for Underwater Sound, Springer, 2007.

[7] J. B. Restorff, M. Wun-Fogle, A. E. Clark, T. A. Lograsso, A. R. Ross, and D. Schlagel, "Magnetostriction of ternary Fe-Ga-X alloys (X=Ni, Mo, Sn, Al)," Journal of Applied Physics, 91(10), pp. 8225-8227, 2002.

[8] P. G. Evans. Nonlinear Magnetomechanical Modeling and Characterization of Galfenol and System-Level Modeling of Galfenol-Based Transducers, Ph.D. thesis, The Ohio State University, Columbus, Ohio, 2009.

[9] T. Ueno, and T. Higuchi. Investigation of Micro Bending Actuator using Iron-Gallium Alloy (Galfenol). in International Symposium on Micro-NanoMechatronics and Human Science, pp. 460-465, 2007.

(C) Qinghua et al.; Licensee Bentham Open.

This is an open access article licensed under the terms of the Creative Commons Attribution Non-Commercial License (http://creativecommons.org/licenses/ by-nc/3.0/) which permits unrestricted, non-commercial use, distribution and reproduction in any medium, provided the work is properly cited. 